\title{
4 Forschungsdesign und Methoden
}

\subsection{Forschungsdesign}

Van Aelst und Walgrave (2011) bemerken, dass die Ergebnisse der Forschung zu Agenda Setting zwischen Parlament und Medien stark davon abhängen, was für Methoden, oder breiter, Forschungsdesigns, gewählt werden. Konkret vergleichen die Autoren die Diskrepanz der Ergebnisse zwischen quantitativen Analysen von Medieninhalten und parlamentarischen Inhalten sowie quantitativen oder qualitativen Befragungen von Parlamentarierinnen und Parlamentariern. Es gibt zwei grobe, generelle Herangehensweisen an diese Problematik. Die erste ist, die Schwierigkeiten mit dem gewählten Design und den dazugehörigen Methoden bewusst anzusprechen und die gewonnenen Ergebnisse entsprechend kritisch zu reflektieren. Die zweite Herangehensweise ist, beide Designs, das auf Inhaltsanalyse und das auf Befragungen fokussierte, in einem einzigen Forschungsprogramm zu vereinen. Für die vorliegende Arbeit habe ich mich für die zweite Herangehensweise entschlossen.

Eine Kombination unterschiedlicher Forschungsdesigns und Methoden zum Zwecke der besseren Erfassung eines Forschungsgegenstandes wird metaphorisch als Triangulation bezeichnet. Die Grundidee von Triangulation ist simpel. Denzin (1970, S. 13) beschreibt Triangulation folgendermassen:

If each method leads to different features of empirical reality, then no single method can ever capture all the relevant features of that reality; consequently, sociologists must learn to employ multiple methods in the analysis of the same empirical events.

Wenn auch die Grundidee von Triangulation simpel ist, herrscht Uneinigkeit darüber, wie genau Triangulation umzusetzen ist. Ein Teil der Uneinigkeit rührt daher, dass nicht immer klar ist, ob und wie sich Triangulation vom Konzept des «Mixed Methods Research» unterscheidet oder unterscheiden soll. Johnson und Onwuegbuzie (2004) bemerken in einem Übersichtsartikel, dass es keine einheitliche Definition von Mixed Methods Research gibt und sie schlagen darum eine solche vor (ebd., S. 132):

Mixed methods research is the type of research in which a researcher or team of researchers combines elements of qualitative and quantitative research approaches (e.g., use of qualitative and quantitative viewpoints, data collection, analysis, inference techniques) for the broad purposes of breadth and depth of understanding and corroboration. 
Eine solche breite Definition ist wohl prinzipiell kompatibel mit der Idee der Triangulation. So gibt es denn auch Autoren, welche Triangulation als die gleichzeitige Verwendung qualitativer und quantitiativer Methoden definieren (Jick 1979). Das ursprüngliche Verständnis von Triangulation zielt aber nicht auf das Kombinieren quantitativer und qualitativer Methoden ab, sondern auf das Kombinieren unterschiedlicher qualitativer Methoden (Denzin 2012). Mixed Methods Research hat den Ursprung bzw. die häufigste Verwendung im Gesundheitsbereich (Sale, Lohfeld und Brazil 2002). Ein typisches Design in einem solchen Kontext ist eine qualitative Vorstudie für eine quantitative Hauptstudie, oder allenfalls eine qualitative «Nachstudie» nach der quantitativen Hauptstudie. Mixed Methods Research hat schwerpunktmässig somit zwei Haupteigenschaften: Der qualitative und der quantitative Teil stehen in einem sequentiellen Verhältnis, und der Hauptfokus liegt auf dem quantitativen Teil. Flick u. a. (2012) erachten Mixed Methods Reasearch und Triangulation gemäss der angesichts der unterschiedlichen dahinterstehenden Konzepte als zwar prinzipiell separat, aber als letztlich doch vereinbar: Triangulation hat zum Ziel, denselben Gegenstand mit unterschiedlichen qualitativen Methoden zu untersuchen, während Mixed Methods Research quantitative und qualitative Methoden kombiniert, aber nicht denselben Gegenstand trianguliert. Es gibt a priori keinen Grund, warum diese zwei Prinzipien nicht kombiniert werden können, indem also mit qualitativen und quantitativen Methoden derselbe Gegenstand untersucht wird. Wenn ein Mixed Methods-Design als Triangulation umgesetzt wird, ändern sich diese Haupteigenschaften: Der qualitative und der quantitative Teil stehen in einem parallelen Verhältnis - derselbe Gegenstand wird untersucht -, und die qualitativen und quantitativen Daten werden als gleich bedeutsam in Betreff ihrer Aussagekraft betrachtet ${ }^{26}$. Das ist das Design der vorliegenden Arbeit.

Leech und Onwuegbuzie (2007) haben eine Typologie innerhalb der Mixed MethodsDenkschule erarbeitet, mit der das verwendete Design in dieser Arbeit genauer beschrieben werden kann. Die Autoren unterscheiden nach acht Mixed Methods-Designs, welche sich anhand von drei Dimensionen unterscheiden: Der «Mixing»-, der zeitlichen und der «Betonungs»Dimension. Für die «Mixing»-Dimension unterscheiden die Autoren nach teilweisen und vollen Mixed Methods. Teilweise Mixed Methods sind Designs, bei denen es einen qualitativen und einen quantitativen Teil gibt, wobei die Datenerhebung und die Datenauswertung jeweils zwei Mal qualitativ und zwei Mal quantitativ ist. Als volle Mixed Methods betrachten die

26 Howe (2012) beschreibt den Unterschied zwischen einem sequentiellen und einem parallelen Verhältnis als Unterschied zwischen einer «disjunctive», also trennenden, und einer «conjunctive», also verbindenden Anwendung von Triangulation. Eine solche Unterscheidung scheint mir weniger präzise als die Unterscheidung nach sequentiell und parallel; nicht zuletzt, weil die Metapher der Triangulation impliziert, dass Triangulation eben darum zustande kommt, weil die Methoden in einem parallelen Verhältnis stehen. Eine Triangulation, bei der die Methoden «disjunct», also getrennt sind, sorgt bloss für definitorische Verwirrung. 
Autoren Designs, bei denen es auch zwischen Datenerhebung und Datenauswertung einen Methodenwechsel gibt, z.B., indem qualitative erhobene Daten quantitativ ausgewertet werden. Die Zeitdimension betrifft die Frage, ob der qualitative und der quantitative Teil in einem sequentiellen oder in einem parallelen Verhältnis stehen - und die Zeitdimension ist letztlich entscheidend für die Klassifikation eines Designs als Triangulation; nur paralleles Vorgehen ist wirkliche Triangulation im engeren Sinn. Die Betonungs-Dimension schliesslich ist der bereits erwähnte Aspekt, dass es zu entscheiden gilt, welchem Teil des Designs, dem qualitativen oder dem quantitativen, in der Untersuchung mehr Aussagekraft und Bedeutung zugesprochen wird. Die Eigenschaften des Designs dieser Arbeit sind ein volles Mixing, ein paralleles Verhältnis der qualitativen und quantitativen Teile sowie eine gleichwertige Bedeutung der qualitativen und quantitativen Ergebnisse. Die detaillierten Aspekte des Designs sind in den nachfolgenden Abschnitten 4.2 bis 4.4 besprochen.

\subsection{Datenerhebung}

Die Datenerhebung besteht aus zwei separaten Teilen: Der Inhaltsanalyse von Medien und Parlamentsprotokollen zum einen, Leitfadeninterviews zum anderen. Das Vorgehen für die Inhaltsanalyse ist in Unterunterabschnitt 4.2.1 beschreiben, das Vorgehen für die Leitfadeninterviews in Unterunterabschnitt 4.2.2.

\subsubsection{Inhaltsanalyse}

Das Ziel der Inhaltsanalyse in der vorliegenden Arbeit ist es, zutage zu fördern, welche konkreten Themen wann und wie oft in welchen parlamentarischen Arenen sowie in den Medien vorkommen, sowie, zu welchen Politikfeldern die einzelnen konkreten Themen in den parlamentarischen Arenen und in den Medien zugehörig sind. Mit dieser knappen Beschreibung ist im Grunde erschöpfend aufgezählt, was für Variablen erhoben werden, nämlich:

- Konkretes Thema der Untersuchungseinheit

- Politikfeld der Untersuchungseinheit

- Erhebungseinheit (Wo)

- Datum der Erhebungseinheit (Wann)

Die Anzahl der inhaltsanalytisch erhobenen Variablen ist also sehr überschaubar, aber nicht ganz trivial. Ein zentrale Entscheidung für die Inhaltsanalyse betrifft nämlich die Frage, welche Inhalte des Parlamentes und welche Inhalte der Medienstichprobe genau untersucht werden 
sollen - was also die Erhebungs- und die Untersuchungseinheiten sein sollen. In Unterabschnitt 3.1 beschreibe ich, dass sich die Agenda Setting-Forschung in erster Linie der Frage der Themen widmet. Darum fokussiere ich auch bei der Inhaltsanalyse auf diese direkte inhaltliche Komponente und nicht auf erweiterte Aspekte, z.B. die Frage, wie genau die Themen präsentiert werden. Für die Inhaltsanalyse sind zwei Kernvariablen erfasst: Die konkreten Themen sowie die Politikfelder. Diese zwei Variablen, das jeweilige Thema und das jeweilige Politikfeld einer Untersuchungseinheit, sind ähnliche Variablen, aber nicht identische. Bevor ich aber erkläre, was die konkreten Themen und was die Politikfelder sind, ist ein Verweis auf Tabelle 2 angebracht. In Tabelle 2 ist zusammengefasst, was die Erhebungs- und was die Untersuchungseinheiten für die Kernvariablen Thema und Politikfeld für die unterschiedlichen Quellen sind.

Tabelle 2: Erhebungs- und Untersuchungseinheiten für die Kernvariablen Thema und Politikfeld.

\begin{tabular}{lll}
\hline & Erhebungseinheit & Untersuchungseinheit \\
\hline Zeitungen & Zeitungsausgabe & Zeitungsartikel \\
Fernsehen & Einzelne Sendung & Beitrag innerhalb der Sendung \\
Radio & Einzelne Sendung & Beitrag innerhalb der Sendung \\
Parlament: Geschäfte NR & Tagesdebatte & Wortmeldung \\
Parlament: Geschäfte SR & Tagesdebatte & Wortmeldung \\
Parlament: Vorstösse & Eingereichte Vorstösse pro Tag & Vorstoss \\
Parlament: Fragestunden NR & Fragestunde & Wortmeldung \\
Parlament: Abstimmungen NR & Geschäft & Geschäft \\
Parlament: Abstimmungen SR & Geschäft & Geschäft \\
\hline
\end{tabular}

Für die Medien sind die Erhebungseinheiten einzelne Zeitungsausgaben bzw. einzelne Sendungen. Die Untersuchungseinheiten für Medien sind einzelne Artikel in Zeitungen und einzelne Beiträge in Sendungen. Die Erhebungseinheit, welche auf Seiten der Medien von Interesse ist, ist also der Korpus der Berichterstattung des jeweiligen Mediums an einem Tag. Die Erhebungsund Untersuchungseinheiten auf Seiten das Parlamentes sind weitgehend in derselben Logik festgelegt: Bei den traktandierten Geschäften des National- und des Ständerates ist die Erhebungsheinheit die an diesem Tag geleistete Arbeit, also die Tagesdebatte, und die Untersuchungseinheiten sind einzelne Wortmeldungen. Für Vorstösse ist die Erhebungseinheit die Gesamtheit der an einem Tag eingereichten Vorstösse und die Untersuchungseinheit der einzelne Vorstoss. Bei Fragestunden sind, analog dazu, die gesamten Fragestunden die Erhebungseinheiten und die einzelnen Wortmeldungen die Untersuchungseinheiten ${ }^{27}$. Einzig bei den Abstimmungen im National- und im Ständerat sieht es anders aus: Hier sind sowohl die

\footnotetext{
27 Die meisten Fragen in den Fragestunden bestehen aus zwei Wortmeldungen: Der Frage einer Nationalrätin oder eines Nationalrates und der Antwort eines Bundesratsmitglieds.
} 
Erhebungs- wie auch die Untersuchungseinheiten einzelne Geschäfte. Für Abstimmungen würde es, logischerweise, keinen Sinn machen, diese nach einzelnen Wortmeldungen zu erfassen, denn Abstimmungen erfolgen auf der Ebene von Geschäften. Bei Abstimmungen wird also lediglich erfasst, wie viele Ja- und Nein-Stimmen es gibt. Die Ebene der Geschäfte ist praktischerweise auch für die anderen parlamentarischen Untersuchungseinheiten relevant: Alle Wortmeldungen zu einem Geschäft sowie alle Wortmeldungen zu einer Frage in der Fragestunde haben automatisch dasselbe Thema und dasselbe Politikfeld. Dieser Automatismus kommt darum zustande, weil unterschiedliche Wortmeldungen zu einem einzelnen Geschäft alle dieses eine Geschäft betreffen. Das klingt ein wenig verworren, meint aber ganz etwas Banales: Bei einem Geschäft im Parlament kann es mehrere Wortmeldungen geben.

Was genau ist unter konkreten Themen und Politikfeldern zu verstehen? Seit der Studie von McCombs und Shaw (1972) besteht das übliche Vorgehen in der Agenda Setting-Forschung darin, die zu vergleichenden Agenden deduktiv als «Issues» zu bestimmen. Diese Issues befinden sich dabei in einer erkenntnistheoretisch eigentümlichen Schwebe zwischen spezifischen Themen und allgemeinen Politikfeldern. McCombs und Shaw (ebd.) etwa arbeiten mit den Issues «Foreign policy», «Law and Order», «Fiscal policy», «Public welfare», «Civil rights». Andere Studien arbeiten mit anderen Issues. Beispielsweise operationalisieren Shehata und Strömbäck (2013) Agenden nach den Issues «Unemployment», «Health insurance», «Integration/immigration», «Education», «Elderly care», «Environment», «Taxes», «Health care», «Crime». Keine zwei Studien arbeiten mit denselben Issues; es lassen sich auch kurios anmutende Issues finden, wie etwa «value politics (such as immigration and foreign aid)» bei Hopmann, Vreese und Albaek (2011), bei denen komplett unklar ist, wie sie zustande kommen. Der gängige Umgang mit Issues in Agenda Setting-Forschung ist ein gewichtiges, aber vollends unbeachtetes Problem. Wenn Issues deduktiv a priori definiert werden, um sie in qualitativer Inhaltsanalyse in Agenden zu suchen, dann müsste die Herleitung dieser Issues begründbar sein. Diese Diskussion fehlt aber in der Agenda Setting-Forschung, und die Natur der Issues und deren erkenntnistheoretischer Stand wird ignoriert - der Umgang mit Issues in der Agenda Setting-Forschung ist weitgehend von einer ad hoc-Herangehensweise geprägt. ${ }^{28}$. Ich verzichte auf den Einsatz von Issues, weil sie zu beliebig, da nicht genau und präzise begründbar sind. Stattdessen verwende ich für die Inhaltsanalyse einerseits generalisierte Politikfelder und andererseits spezifische Themen der Untersuchungseinheiten.

$\overline{28} \quad$ Die einzige Ausnahme bildet der Versuch, Issues danach zu unterscheiden, ob sie für gewöhnliche Bürgerinnen und Bürger im Alltag direkt spürbar und von Belang sind («obtrusive» Issues), oder, ob die Issues für sie eher abstrakt bleiben und keinen Bezug zum Alltag haben («unobtrusive» Issues) (Demers u. a. 1989). 
Wenn Politik in den drei Dimensionen Politics (politische Prozesse), Polity (politische Strukturen) und Policy (politische Inhalte) gedacht wird, dann ergeben sich Politikfelder aus dem Versuch, die konkreten Inhalte von Politik nach Bereichen zu bündeln. Politikfelder sind letzlich aber induktive Konstrukte, welche nicht immer trennscharf sind (Blum 2011, S. 14-17). Dennoch ist die Definition von Politikfeldern in einem genüngenden Masse ausgereift, dass es möglich ist - anders als bei Issues - auf eine nicht-willkürliche Kategorisierung zurückzugreifen. Für den in dieser Arbeit verwendeten Katalog an Politikfeldern stütze ich mich auf die Einteilung nach Knoepfel u. a. (2014, S. 623-911). Die verwendeten Politikfelder für die Inhaltsanalyse sind in Tabelle 3 zusammengetragen. Nebst den Politikfeldern sind in Tabelle 3 auch die Politikdimensionen Politics und Polity vermerkt. Diese Politikdimensionen sind zwar keine Politikfelder, aber im Laufe der Erarbeitung des inhaltsanalytischen Vorgehens hat es sich als sinnvoll erwiesen, auch diese zwei zusätzliche Variablen mitzuerfassen. Medien berichten auch über politische Prozesse ohne spezifischen Bezug zu politischen Inhalten (etwa bei personellen Veränderungen bei Parteien, oder bei Analysen von Abstimmungsverhalten, und so fort) wie auch über politische Strukturen, ebenfalls ohne Bezug zu konkreten politischen Inhalten (etwa in Analysen zu Vor- und Nachteilen direkter Demokratie, und so fort). Darüber hinaus können auch parlamentarische Inhalte Bezug zu Politics oder zu Polity und weniger zu konkreten Politikfeldern haben. Von der Logik her stellen die einzelnen Politikfelder das Spektrum der Politikdimension Policy dar. Darum wurde Policy nicht nochmals als separate Politikdimension erfasst.

Wenn auch die Politikfelder deduktiv vorgegeben sind, ist die inhaltsanalytische Arbeit mit ihnen nicht trivial. Die Zuweisung einzelner Politikfelder zu den in Tabelle 2 zusammengefassten Untersuchungseinheiten ist ein qualitativer, sinnverstehender Vorgang, der aber systematisiert werden muss. Die systematische Zuweisung deduktiver Kategorien, wie sie für diese Inhaltsanalyse vorgenommen wurde, kann nach Mayring (2010) als strukturierende Inhaltsanalyse bezeichnet werden. Die genaue Bezeichnung für diese Art der deduktiven qualitativen Inhaltsanalyse ist aber nicht einheitlich; Hsieh und Shannon (2005) beispielsweise nennen dieses deduktive Vorgehen «directed content analysis». Wie auch immer die Bezeichnung ausfällt, das Vorgehen bleibt dasselbe: Die qualitative Kategorisierung des Materials erfolgt anhand expliziter Regeln und idealerweise durch mehrere Personen. Für diese Analyse hat ein Team von drei Personen zuerst gemeinsam die Analyseregeln geübt und anschliessend die Inhaltsanalyse durchgeführt. Die Intercoder-Reliabilität anhand des Krippendorff Alpha-Wertes bewegt sich dabei im Bereich von 0.7, was akzeptabel ist (Krippendorff 2004). Dieser Wert repräsentiert allerdings nur eine Trockenübung und nicht das tatsächliche Vorgehen im Inhaltsanalyse-Team. 
Tabelle 3: Liste der verwendeten Politikfelder, in alphabetischer Reihenfolge.

\begin{tabular}{ll}
\hline Politikfelder & Aussenpolitik \\
& Äussere Sicherheit \\
& Bildungspolitik \\
& Einwanderungs- und Asylpolitik \\
& Finanzpolitik \\
& Forschungspolitik \\
& Gesundheitspolitik \\
& Infrastrukturpolitik \\
& Innere Sicherheit \\
& Internationale Politik \\
& Kulturpolitik \\
& Landwirtschaftspolitik \\
& Medienpolitik \\
& Sozialpolitik \\
& Umwelt- und Raumordnungspolitik \\
& Wirtschaftspolitik \\
\hline Politikdimensionen & Politics \\
& Polity \\
\hline
\end{tabular}

Bei einer Trockenübung weist jede Person separat Kategorien zu, unabhängig davon, wie sicher oder unsicher sie ist. Bei der effektiven Inhaltsanalyse haben wir hochgradig kollaborativ gearbeitet: Nebst regelmässigen Treffen mit Besprechungen von Problemfällen und dergleichen haben wir mit einem interaktiven Online-Codiersystem steten Austausch gepflegt. Damit war es u.a. möglich, bei Unklarheiten und Problemfällen die anderen Teammitglieder in Echtzeit zu benachrichtigen, um eine gemeinsame Lösung zu bestimmen. Dadurch fällt die Reliabilität der deduktiven Inhaltsanalyse höher aus als ein Test auf Intercoder-Reliabilität ohne diese kollaborative Komponente suggeriert.

Der zweite Teil der Inhaltsanalyse ist das induktive bestimmen der spezifischen Themen der Untersuchungseinheiten. Ein induktives Vorgehen stellt andere Anforderungen als ein deduktives, denn die zuzuweisenden Kategorien sind nicht a priori definiert, sondern werden aus den Inhalten herausgearbeitet. Das Herausarbeiten der Themen der Untersuchungseinheiten als Form der qualitativen Inhaltsanalyse wird in der Literatur auf unterschiedliche Art beschrieben. Hsieh und Shannon (2005) nennen dieses Vorgehen «conventional content analysis», weil, so die Autoren, diese Form der qualitativen Inhaltsanalyse die zentrale Form qualitativer Inhaltsanalyse darstellt. Mayring (2010) beschreibt diese Form der Inhaltsanalyse als zusammenfassende Inhaltsanalyse. Der Arbeitsablauf bei dieser zusammenfassenden Inhaltsanalyse ist aber nicht komplett induktiv, sondern auch iterativ-deduktiv. Das bedeutet 
für die durchgeführte Inhaltsanalyse konkret, dass die thematische Zusammenfassung einer Untersuchungseinheit in einem ersten Schritt qualitativ-induktiv stattfindet. Die durch diese induktive Zusammenfassung definierte Kategorie wird anschliessend - und das ist ein zentraler Schritt - mit einer Datenbank aller definierten Kategorien abgeglichen. Falls bisher keine Einträge in diesem Thementopf vorhanden sind, welche die induktiv definierte neue Kategorie bereits abdecken, dann wird der Thementopf um diese neue Kategorie erweitert. Falls aber bereits eine Kategorie im Thementopf vorhanden ist, welche die induktiv definierte neue Kategorie bereits abdeckt, dann wird die im Thementopf bereits vorhandene Kategorie verwendet. Das grundlegende Vorgehen für die qualitative Bestimmung der konkreten Themen ist also für jede einzelne Untersuchungseinheit induktiv, die einzelnen induktiven Arbeitsschritte werden aber darüber hinaus zusätzlich iterativ-deduktiv angereichert. Diese Form der qualitativen Inhaltsanalyse wird im Englischen bisweilen als «thematic analysis» beschrieben (Braun und Clarke 2006; Fereday und Muir-Cochrane 2008). Der Vorteil eines solchen kombinierten induktiven und iterativ-deduktiven Vorgehens ist, dass die Kategorisierung jeder einzelnen Untersuchungseinheit sinngeleitet und reflektiert ist. Für jede Untersuchungseinheit fallen zwei kognitiv anspruchsvolle Schritte an, die induktive Zusammenfassung und der anschliessende Abgleich des Ergebnisses dieser Zusammenfassung mit dem iterativ erarbeiteten Thementopf. Die Kehrseite dieses Vorgehens ist der Aufwand: Der Prozess des induktiven Zusammenfassens, des Konsultierens und ggf. Erweiterns des Thementopfes sowie das häufige und ausführliche Diskutieren im Team erfordert viel Zeit.

Die Inhalte des Parlamentes werden anhand der Parlamentsprotokolle und der eingereichten Vorstösse in toto analysiert, für die Medienstichprobe erfolgt angesichts der aufwändigen qualitativen Inhaltsanalyse aber eine Einschränkung. Nur jene Zeitungsartikel bzw. Beiträge in Sendungen fliessen in die Inhaltsanalyse, welche folgende Bedingungen erfüllen:

- Es werden ausschliesslich Inhalte der journalistischen, redaktionellen Teile berücksichtigt (Anzeigen, Inserate, Unterhaltendes und dergleichen werden nicht berücksichtigt).

- Bei den journalistischen, redaktionellen Teilen müssen Artikel zwei Bedingungen erfüllen, um für die Inhaltsanalyse berücksichtigt zu werden:

- Die Artikel bzw. Beiträge müssen einen expliziten Bezug zu Politik haben.

- Die Artikel bzw. Beiträge müssen einen expliziten Bezug zur Schweiz haben.

Nachdem definiert ist, was die Erhebungs- und was die Untersuchungseinheiten sind, sowie, wie die konkreten Themen und die Politikfelder erhoben werden, gilt es noch, das Wann zu klären, also die zu untersuchenden Zeiträume zu bestimmen. Da eine Totalerhebung aller 
Parlamentsaktivitäten und aller Berichterstattung zum Parlament keine ernsthafte Option ist, arbeite ich mit Stichproben von Sessionen in der Periode der Medienkrise. Konkret untersuche ich die Aktivitäten der Bundesversammlung und der Medien innerhalb dreier Sessionen. Die Eckpunkte zu den drei ausgewählten Sessionen sind in Tabelle 4 festgehalten.

Tabelle 4: Sessionen für die Inhaltsanalyse.

\begin{tabular}{llll}
\hline Sessionsbeginn & Sessionsende & Sessionstage & Sessionstyp \\
\hline 20.09 .1999 & 08.10 .1999 & 13 & Herbstsession \\
19.09 .2005 & 07.10 .2005 & 13 & Herbstsession \\
12.09 .2011 & 30.09 .2011 & 13 & Herbstsession \\
\hline
\end{tabular}

Für die Analyse betrachte ich drei Herbstsessionen, jene von 1999, jene von 2005 und jene von 2011. Jede dieser Sessionen dauert reguläre drei Wochen, bzw. genauer, 13 Tage (In der den ersten zwei Sessionswochen wird von Montag bis Donnerstag getagt, in der dritten Woche von Montag bis Freitag.). 1999 ist ein Jahr, in welchem die Medienkrise noch nicht in vollem Zug ist, sondern sich erst ankündigt. Wie in Abbildung 1 festgehalten ist, sind in dieser Zeit die Werbeeinnahmen bei Printtiteln noch im Aufstieg begriffen, und Pendlerzeitungen sind noch kaum auf dem Markt vertreten ${ }^{29} .1999$ stellt somit einen Zeitpunkt vor der Medienkrise dar, und 2005 und 2011 sind zwei Zeitpunkte, zu denen die Medienkrise jeweils bereits im Gange ist (2005) und sich weiter verschärft (2011). Die Aktivitäten aller drei Sessionen sind über das amtliche Bulletin (Protokolle der Debatten und Abstimmungen sowie der Fragestunden) sowie über Curia Vista (eingereichte Vorstösse) zugänglich. Die Inhaltsanalyse der Parlamentsprotokolle und der Vorstösse erfolgt mittels Totalerhebung; für die drei Sessionen werden also alle Aktivitäten inhaltsanalytisch erfasst.

Der nächste Schritt der Stichprobenbildung betrifft die Tage innerhalb der Sessionen, für welche die noch zu bestimmende Medienstichprobe untersucht werden soll. Eine Minimalvariante diesbezüglich wäre es, die Medienstichprobe nur an genau jenen 13 Tagen pro Session zu ziehen, an denen auch die Bundesversammlung tagt. Eine solche Minimalvariante dürfte aber den journalistischen Umgang mit der Session nicht gerecht werden. Es ist nämlich anzunehmen, dass eine Session am Wochenende vor Sessionsbeginn und am Wochenende nach Sessionsende in den Medien behandelt wird, und, dass während der Session auch an tagungsfreien Tagen über die Session berichtet wird. Darum habe ich den jeweils vollen Sessionszeitraum, inklusive des Wochnendes vor Sessionsbeginn und des Wochenendes nach Sessionsende, als Zeitraum für die Medienstichprobe gewählt, wie in Tabelle 5 zusammengefasst ist.

$29 \quad 20$ Minuten kam im Dezember 1999 als erste Pendlerzeitung auf den Markt. 
Tabelle 5: Zeiträume für die Medienstichproben.

\begin{tabular}{llll}
\hline Beginn & Ende & Tage & Wochentage \\
\hline 18.09 .1999 & 10.10 .1999 & 23 & 15 \\
17.09 .2005 & 09.10 .2005 & 23 & 15 \\
10.09 .2011 & 02.10 .2011 & 23 & 15 \\
\hline
\end{tabular}

Die Auswahl der konkreten Medientitel ist ein heikler Schritt. Mit der konkreten Stichprobe an Medien wollte ich zwei Ziele erreichen. Zunächst habe ich im Vorfeld bestimmt, dass Titel aus Print, Fernsehen und Radio in die Stichprobe einfliessen sollen. Das zweite Kriterium sollte sein, dass nur solche Titel pro Gattung in die Auswahl kommen, welche den Status von Leitmedien haben. Nun gibt es aber keine einheitliche Definition der Kriterien, welche ein Medium zu einem Leitmedium machen. Künzler (2012) argumentiert etwa, dass die Bezeichnung des Leitmediums stark davon abhängt, für welche Zielgruppe das Medium eine leitende funktion haben soll. Pragmatischerweise habe ich solche Titel in die Medienstichprobe aufgenommen, welche in der Deutschschweiz reichweitenstark hinsichtlich politischer Kommunikation sind. Die Stichprobe an Zeitungstiteln und Sendungen ist in Tabelle 6 zusammengefasst.

Tabelle 6: Medienstichprobe pro Jahr, inklusive der Anzahl Zeitungsausgaben bzw. Fernseh- und Radiosendungen pro Stichprobenahr.

\begin{tabular}{llll}
\hline & 1999 & 2005 & 2011 \\
\hline Neue Zürcher Zeitung & 19 & 19 & 19 \\
Tages-Anzeiger & 19 & 19 & 19 \\
Blick & 19 & 19 & 19 \\
20 Minuten & - & 15 & 15 \\
Blick am Abend & - & - & 15 \\
SonntagsBlick & 4 & 4 & 4 \\
SonntagsZeitung & 4 & 4 & 4 \\
NZZ am Sonntag & - & 4 & 4 \\
Echo der Zeit & 15 & 23 & 23 \\
Tagesschau & 23 & 23 & 23 \\
\hline
\end{tabular}

Wenn eine Zelle mit «-» markiert ist, bedeutet das, dass in dem betroffenen Jahr im Zeitraum der Session der betroffene Titel noch nicht existierte. Die Anzahl der Echo der Zeit-Ausgabe ist für 1999 geringer als für 2005 und 2011, weil es 1999 noch keine Wochenendausgaben des Echo der Zeit gab.

Wie in Tabelle 6 ersichtlich ist, ist die Anzahl Zeitungstitel weit grösser als die Anzahl der Fernseh- und Radiosendungen in der Stichprobe; für das Fernsehen ist lediglich die Tagesschau in der Stichprobe, für das Radio lediglich das Echo der Zeit. Das mag auf den ersten Blick als Verzerrung erscheinen, ist in Tat und Wahrheit einigermassen repräsentativ für die Medien- 
landschaft der Schweiz: Bei Fernsehen und Radio werden de facto nur die Kanäle von SRF in der gesamten jeweiligen Sprachregion rezipiert ${ }^{30}$. Die Printzeitungen in der Medienstichprobe sollen eine möglichst grosse Vielfalt aufweisen. Darum sind nebst den Tageszeitungen NZZ, Tages-Anzeiger und Blick auch die Pendlerzeitungen 20 Minuten und Blick am Abend in der Stichprobe, ebenso wie die Sonntagszeitungen SonntagsBlick, SonntagsZeitung und NZZ am Sonntag.

In Tabelle 4 ist zusammengefasst, welche Zeiträume für welche Sessionen erfasst werden, und in Tabelle 6, welche Stichprobentage für die Medienstichprobe. Gemeinsam mit den oben beschriebenen Auswahlkriterien für Artikel in Zeitungen und Sendungsbeiträge in Fernsehen und Radio lässt sich daraus nun die totale resultierende Stichprobe anhand der Anzahl in der Inhaltsanalyse analysierter Untersuchungseinheiten bestimmen. Die Gesamtsrichprobe ist in Tabelle 7 zusammengefasst.

Tabelle 7: Anzahl der für die qualitative Inhaltsanalyse erfassten Untersuchungseinheiten.

\begin{tabular}{llll}
\hline & 1999 & 2005 & 2011 \\
\hline Neue Zürcher Zeitung & 439 & 464 & 596 \\
Tages-Anzeiger & 449 & 460 & 406 \\
Blick & 243 & 193 & 222 \\
20 Minuten & - & 173 & 165 \\
Blick am Abend & - & - & 75 \\
SonntagsBlick & 161 & 110 & 66 \\
SonntagsZeitung & 160 & 123 & 125 \\
NZZ am Sonntag & - & 126 & 177 \\
Echo der Zeit & 146 & 180 & 136 \\
Tagesschau & 156 & 106 & 91 \\
\hline Geschäfte NR & 1113 & 1580 & 1535 \\
Geschäfte SR & 561 & 561 & 695 \\
Fragestunden & 150 & 201 & 210 \\
Vorstösse & 148 & 222 & 316 \\
\hline
\end{tabular}

Wenn eine Zelle mit «-» markiert ist, bedeutet das, dass in dem betroffenen Jahr im Zeitraum der Session der betroffene Titel noch nicht existierte. Die Anzahl Untersuchungseinheiten für den Ständerat sind für 1999 und 2005 bloss aus Zufall gleich.

Die Fallzahlen der finalen Stichprobe für die Medien und die Fallzahlen der Totalerhebung für das Parlament sind, wie in Tabelle 7 zusammengefasst ist, je nach Medium bzw. parlamentari-

30 Ein wenig anders sieht es im Kanton Tessin aus, wo private Radio- und Fernsehstationen einen grossen Teil der Sprachregion erfassen - dies aber nur, weil die italienischsprachige Sprachregion der Schweiz im Wesentlichen nur aus dem Kanton Tessin besteht. Nebst des Tessins wird Italienisch noch in einigen Tälern des Kantons Graubünden dominanterweise gesprochen. 
scher Arena im zweistelligen bis vierstelligen Bereich. Bei dieser Gesamthaft verhältnismässig hohen Fallzahl pro Stichprobenjahr überrascht es nicht, dass auch die Anzahl der erfassten spezifischen Themen verhältnismässig hoch ausfällt: Für 1999 wurden 947 unterschiedliche Themen erfasst, für 2005 deren 1'115 und für 2011 mit 1'252 am meisten. Der Umstand, dass zunehmend mehr Themen erfasst wurden, überrascht nicht, denn 2005 sind mehr Medientitel in der Stichprobe enthalten als 1999, und 2011 nochmals mehr als 2005.

\subsubsection{Leitfadeninterviews}

Die zweite qualitative Datenerhebung als triangulierende Ergänzung der Inhaltsanalyse sind teilstrukturierte Interviews mit Parlamentarierinnen und Parlamentariern sowie mit Journalistinnen und Journalisten. Teilstrukturierte Interviews, oder synonym, Leitfadeninterviews, sind in zweifacher Hinsicht eine sinnvolle Ergänzung der Inhaltsanalyse. Erstens sind die Untersuchungsgegenstände ${ }^{31}$ bei Leitfadeninterviews deutlich andere als bei der Inhaltsanalyse - Triangulation macht dann am meisten Sinn, wenn nicht identisches Datenmatierial mit bloss unterschiedlichen Methoden analysiert wird, sondern, wenn grundlegend unterschiedliches Datenmaterial generiert wird. Zweitens bedeuten Leitfadeninterviews, dass direkt jene Akteure zu Wort kommen, für welche der Gegenstand der vorliegenden Untersuchung nicht ein abstraktes Thema ist, sondern unmittelbarer Teil ihrer Lebenswelt.

Qualitative Interviews können sehr unterschiedliche Formen aufweisen, denen gemein ist, dass, anders als in standardisierten Befragungen, die Interviewpartner prinzipiell offen auf Fragen antworten bzw. frei sprechen und nur ausnahmsweise mit geschlossenen Fragen konfrontiert werden. Es ist dabei üblich, die Fragen für das Interview nicht im Voraus fix zu definieren, sondern auch situativ innerhalb des Gespräches mit (Nach-)Fragen zu reagieren Weiss (1995). Hopf (2004) schlägt vor, die mögliche Vielfalt an qualitativen Interviewmethoden anhand dreier Dimensionen zu typologisieren. Erstens kann die inhaltliche Ausrichtung des Interviews unterschiedlich stark gesteuert werden; eine starke Steuerungsmöglichkeit ist beispielsweise eine rigide Katalogisierung von Fragen, welche in einer bestimmten Reihenfolge im Interview beantwortet werden müssen. Zweitens kann der Gegenstand des Gespräches sehr fokussiert bis sehr breit sein. Sehr fokussierte Gesprächsgegenstände könne beispielsweise Gespräche sein, bei denen ein spezifischer Filmausschnitt besprochen wird. Drittens gilt es zu entscheiden, ob das Ziel des Interviews eher auf aktivem Fragen seitens der Forscherin oder des Forschers liegen soll, oder eher das Ziel ist, dass die Forscherin oder der Forscher eine

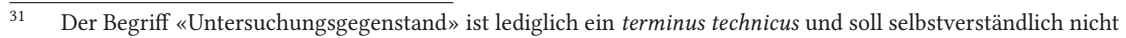
bedeuten, dass meine Gesprächspartnerinnen und -partner Gegenstände sind. 
passive Rolle einnimmt und die Interviewpartner von sich aus aktiv und frei erzählen. Wenn auch diese drei Dimensionen nicht ganz unabhängig voneinander sind, ist das Argument, dass qualitative Interviews ein Spektrum von gesteuert und rigide bis offen und flexibel einnehmen können, wichtig. Hopf (ebd., S. 204) bemerkt, dass eine bestimmte Form der Interviews, welche eine mittlere Position in diesem Spektrum einnimmt, besonders häufig zum Einsatz kommt:

The variants of qualitative interviews that are used particularly frequently are between these extremes and may be described as relatively flexibly applied semistandardized interviews: researchers orient themselves according to an interview guide, but one that gives plenty of freedom of movement in the formulation of questions, followup strategies and sequencing.

Dise sogenannten halbstrukturierten Interviews verbinden einen verhältnismässig hohen Grad der Strukturiertheit Strukturiertheit bezüglich der Diskussionsziele mit der Flexibilität, im Verlaufe des Gespräches situativ zu reagieren und wenn nötig zu vertiefen. Flick (2014, S. 227) schlägt vor, teilstrukturierte Interviews, welche nicht auf die allgemeine Biographie der Interviewpartner abzielen, sondern auf das Fachwissen der Interviewpartner in einem bestimmten Bereich, als Experten- oder Elite-Interviews zu verstehen. Die Gespräche, welche ich für die vorliegende Arbeit durchgeführt habe, fallen in diesen Typus von Interviews: Sinn und Zweck der Interviews ist es, empirische Einsichten zum professionellen Handeln von Parlamentarierinnen und Parlamentariern sowie von Journalistinnen und Journalisten zu erlangen. Teilstrukturierte Interviews im Sinne von Experteninterviews sind gerade im Bereich der - um einen breiten und vielleicht nicht sehr präzisen Begriff zu verwenden - qualitativen Medienforschung sehr verbreitet und erprobt (Riesmeyer 2011).

Für die Interviews habe ich zwischen Juni 2014 und Dezember 2014 insgesamt 26 Gespräche geführt. Die Verteilung der Interviews auf Parlamentarierinnen und Parlamentarier sowie Journalistinnen und Journalisten ist in Tabelle 8 zusammengefasst.

Um die Anonymität der Journalistinnen und Journalisten zu wahren, spezifiziere ich in Tabelle 8 nicht die Zugehörigkeiten zu einzelnen Medien. Als Population für potenzielle Interviewpartnerinnen und -partner kamen nur jene Journalistinnen und Journalisten in Frage, welche für die Medien, welche in der Stichprobe für die Inhaltsanalyse vorkommen, als Bundeshauskorrespondentinnen und -korrespondenten arbeiteten. Auf Seiten des Parlamentes ist die parteiliche Durchmischung befriedigend, wie in Tabelle 9 festgehalten ist. Die Beschreibung «FDP = FDP.Die Liberalen» mag eigentümlich erscheinen, da es eine rekursive Namensauf-
lösung ist. Die Freisinnig-Demokratische Partei (FDP) ist 2009 mit der kleineren Liberalen Partei der Schweiz 
Tabelle 8: Anzahl und Zugehörigkeiten der Interviewpartnerinnen und -partner.

\begin{tabular}{ll}
\hline Zugehörigkeit & Anzahl \\
\hline Nationalrat & 11 \\
Ständerat & 7 \\
Zeitungen & 5 \\
Rundfunk & 3 \\
\hline
\end{tabular}

Bemerkung: Die drei Personen in der Kategorie Rundfunk sind angehörige des öffentlichen Deutschschweizer Rundfunks SRF, bestehend aus Radio und Fernsehen.

Tabelle 9: Parteizugehörigkeiten der interviewten Mitglieder von National- und Ständerat.

\begin{tabular}{ll}
\hline Partei & Anzahl \\
\hline CVP & 5 \\
SP & 5 \\
SVP & 4 \\
FDP & 3 \\
GLP & 1 \\
\hline
\end{tabular}

Legende: CVP = Christlichdemokratische Volkspartei der Schweiz; SP = Sozialdemokratische Partei der Schweiz; SVP = Schweizerische Volkspartei; FDP = FDP.Die Liberalen ${ }^{32}$; GLP = Grünliberale Partei.

Die parteiliche Durchmischung der Interviewpartnerinnen und Interviewpartner seitens des Parlamentes ist zwar befriedigend, aber nicht optimal: Die Parteien sind nicht repräsentativ gemäss ihren Anteilen im National- und Ständerat vertreten (dazu wären grundsätzlich mehr Interviews nötig gewesen), und einige relevante Parteien, etwa die Grüne Partei der Schweiz oder die Bürgerlich-Demokratische Partei, fehlen gänzlich. Aus diesem Grund sind die Interviews nicht geeignet, Aussagen über Gemeinsamkeiten oder Unterschiede der einzelnen Parteien zu machen. Ein letzter Aspekt der Zusammensetzung der Interviewpartnerinnen und Interviewpartner, die Verteilung der Geschlechter, ist in Tabelle 10 zusammegefasst.

Tabelle 10: Geschlechterverteilung der Interviewpartnerinnen und -partner.

\begin{tabular}{lll}
\hline & Frauen & Männer \\
\hline Parlament & 5 & 13 \\
Medien & 4 & 4 \\
\hline
\end{tabular}

In der Stichprobe der Parlamentarierinnen und Parlamentarier sind deutlich mehr Männer als Frauen vertreten. Der Anteil der Gesprächspartnerinnen liegt aber mit knapp 28\% sehr nahe 
am durchschnittlichen Frauenanteil in der Bundesversammlung; 2014 waren im Nationalrat knapp 30\% der Mitglieder Frauen, im Ständerat knapp 20\%. Ich habe unter Einwilligung aller Gesprächspartnerinnen und Gesprächspartner die Interviews als digitale Audiodatei aufgezeichnet. Die aufgezeichneten Interviews wurden anschliessend in einem Team von vier Personen (mich eingeschlossen) zu geschriebenem Text transkribiert. Bei der Transkription von Tonaufnahmen zu Text gibt es unterschiedliche Konventionen. Es gilt beispielsweise, abzuwägen, ob die ganzen Gespräche oder nur bestimmte Ausschnitte transkribiert werden, oder auch, ob wortwörtlich oder leicht paraphrasierend transkribiert wird. Für die Transkription habe ich die Vorschläge von McLellan, MacQueen und Neidig (2003) umgesetzt: Volle Transkription unter Beibehaltung der morphologischen Eigenheiten und syntaktischen Strukturen. Ein solches Vorgehen hat den Vorteil, dass bei der Umwandlung von Ton zu Text so wenig Information wie möglich verloren geht. Der Nachteil ist der hohe zeitliche Aufwand für dieses detaillierte Transkribieren. Bei fünf Interviews wurde mit der Transkribierung zusätzlich eine «Übersetzung» vorgenommen: Fünf Gespräche fanden auf Wunsch der betroffenen Interviewpartnerinnen und -partner in schweizerdeutschem Mundart statt. Diese Gespräche wurden bei der Transkription in Standarddeutsch überführt.

Die Leitfäden für die Interviews sowohl mit Mitgliedern der Bundesversammlung wie auch mit Journalistinnen und Journalisten decken sechs Themenblöcke ab:

1. Präsenz von Medien während der Session allgemein.

2. Umgang mit Medien.

3. Agenda Setting allgemein.

4. Strategisches Handeln.

5. Entwicklung.

6. Normative Bewertung.

Diese Themenblöcke sind inhaltlich nicht trennscharf und in den konkreten Gesprächen bezüglich der Reihenfolge und des Umfangs unterschiedlich. Der erste Themenblock, Präsenz von Medien während der Session allgemein, ist bei allen Gesprächen der sogenannte «Eisbrecher»: $\mathrm{Zu}$ Beginn sollten alle Interviewpartnerinnen und Interviewpartner darüber berichten, wie sie die Präsenz von Journalistinnen und Journalisten während der Session erleben. Bei den Interviews mit Journalistinnen und Journalisten haben diese, logischerweise, über ihren eigenen Berufsalltag berichtet. Der erste Themenblock diente nicht zuletzt dazu, Anknüpfungspunkte für Fragen hinsichtlich der anderen Themenblöcke zu finden. Der zweite Themenblock, Umgang mit Medien, übt vor allem die Funktion der kognitiven Aktivierung aus, durchaus 
im Sinne von Priming (vgl. Unterabschnitt 3.1). Für diesen Themenblock wollte ich von den Parlamentarierinnen und Parlamentariern erfahren, wie sie in ihrer politischen Biographie gelernt haben, mit Medien umzugehen. Die interviewten Journalistinnen und Journalisten sollten hier nicht über sich selber berichten, sondern die Thematik der medialen Erfahrung der Parlamentarierinnen und Parlamentariern aus ihrer Sicht einschätzen, ebenfalls zum Zwecke der kognitiven Aktivierung. Für den dritten Themenblock, Agenda Setting allgemein, bat ich die Interviewpartnerinnen und -partner um ihre Einschätzungen hinsichtlich zweier Agenda Setting-Ströme: Die Auswahl von Themen für die Berichterstattung seitens der Medien sowie die allfällige Beeinflussung des Parlamentes durch die Medien. Für die Auswahl von Themen für die Berichterstattung wurde in den Interviews nach den drei Teilarenen der regulären Geschäfte, der Vorstösse und der Fragestunden unterschieden. Für den allfälligen Einfluss von Medien haben die Gesprächsparterinnen und -partner über dieselben Teilarenen reflektiert, mit dem Unterschied, dass bei regulären Geschäften die Themen institutionellerweise nicht gesetzt, aber eventuell Abstimmungen durch Berichterstattung beeinflusst werden können. Bei diesem Themenblock dreht sich das Gespräch nicht nur darum, $o b$ es solche Beeinflussungen gibt, sondern auch darum, warum es die Beeinflussungen gibt. Der vierte Themenblock, Strategisches Handeln, fokussiert auf die Thematik, was Parlamentarierinnen und Parlamentarier mit Medienpräsenz erreichen wollen sowie, was Journalistinnen und Journalisten mit Berichterstattung über das Parlament erreichen wollen. Beide Akteursgruppen reflektieren dabei ihre eigene Motivstruktur und jene der jeweils anderen Akteursgruppe. Bei dem Themenblock der Entwicklung geht es für alle Interviewpartnerinnen und -partner um die Frage, ob sich rund um die im Laufe des Gespräches an- und besprochenen Dinge etwas verändert hat in den letzten 10 bis 15 Jahren. In dem Themenblock Normative Bewertung schliesslich treffen die Interviewpartnerinnen und -partner Einschätzungen rund um die Frage, ob es, erstens, aus ihrer Sicht Aspekte in dem besprochenen Themenkomplex gibt, welche aus einer demokratischen Perspektive problematisch sind und wie sie, zweitens, die Gesamtsituation des Verhältnisses von Parlament und Medien aus einer demokratischen Perspektive einschätzen.

Nicht alle Themenblöcke der Interviews haben direkten Bezug zu den Forschungsfragen und Hypothesen. Die ersten zwei Themenblöcke haben, wie beschrieben, vor allem eine aktivierende Funktion: Die Interviewpartnerinnen und -partner werden angeregt, sich aktiv in die Wechselwirkungen von Parlament und Medien hineinzuversetzen. Der letzte Themenblock, die normative Beurteilung, ist ebenfalls nicht direkt an die Forschungsfragen und Hypothesen gekoppelt. Die normative Einschätzung ist Teil der Interviews, um jenseits der empirischen Fragestellungen zusätzlich auf einer Metaebene zu reflektieren, wie nah oder wie fern das in 
Unterunterabschnitt 2.2.2 beschriebene normative Modell der prozeduralisierten Volkssouveränität an den normativen Modellen der Parlamentarierinnen und Parlamentarier sowie der Journalistinnen und Journalisten ist (vgl. dazu Unterunterabschnitt 6.2.1).

\subsection{Datenauswertung}

Die Auswertung der Daten erfolgt analog zur Erhebung der Daten in zwei getrennten Schritten. Die mittels qualitativer Inhaltsanalyse erhobenen Daten werte ich mittels quantitativer statistischer Verfahren aus, die Transkripte der teilstrukturierten Interviews hingegen qualitativ. Die genauere Vorgehensart beschreibe ich in den nachfolgenden Unerabschnitten 4.3.1 und 4.3.2. In Unterunterabschnitt 4.3.3 beschreibe ich schliesslich noch kurz, wie die Ergebnisse der separaten Auswertungen für die Besprechung der Ergebnisse zusammengeführt werden.

\subsubsection{Auswertung der Inhaltsanalyse}

Für die quantitative Auswertung der inhaltsanalytisch erfassten Daten bediene ich mich zweier statistischer Verfahren. Zum einen modelliere ich einfache lineare OLS-Regressionen, zum anderen lineare, auf OLS basierende Zeitreihen-Modelle. Das Modell der einfachen OLSRegressionen, welche ich modelliere, hat folgende Form (in Matrix-Notation):

$$
Y=X \beta+\varepsilon
$$

Analog dazu modelliere ich Zeitreihen mit der folgenden Form:

$$
Y_{i, t}=X_{i, t} \beta+\varepsilon_{i, t} ; i=1, \ldots, N ; t=1, \ldots, T
$$

Die so modellierte Zeitreihe ist ein gepooltes OLS-Modell. Auf den ersten Blick mag es überraschen, dass ich keine «konventionelleren» Zeitreihen modelliere, wie etwa ARIMA- oder VAR-Modelle (Hyndman und Athanasopoulos 2013, S. 213-258, 272-275). Der Grund ist die Komplexität der Struktur der inhaltsanalytisch erhobenen Daten. Einfache Zeitreihen sind «zweidimensional»: Sie bestehen aus einer oder mehreren Variablen, deren Werte für unterschiedliche Zeitpunkte im Datensatz enthalten sind. Die Zeitreihe, welche ich verwende, ist aber «dreidimensional»: Nach wie vor werden eine odere mehrere Variablen für unterschiedliche Zeitpunkte erfasst. Diese Variablen werden aber zusätzlich noch für unterschiedliche Einheiten erfasst. Die Daten, mit denen ich für die Zeitreihen arbeite, sind also sogenannte «time-series cross-section»-Daten (Beck 2001). Die Beschreibung dieser Datenstruktur als «time-series cross-section» veranschaulicht die Struktur der Daten. Es handelt sich um Querschnittsdaten, 
welche für mehr als einen Zeitpunkt erhoben werden. Time-series cross-section-, oder abgekürzt, TSCS-Daten sind gängig in der Forschung auf ländervergleichender Ebene, und in diesem Kontext kann gut nachvollzogen werden, was für eine Struktur TSCS-Daten haben. Wenn wir in einem fiktiven Beispiel für die Schweiz, für Deutschland und für die USA vergleichen, wie hoch das BIP und wie hoch die Lebenserwartung im Jahr 2014 war, dann handelt es sich um einen Querschnitt. Wenn wir aber vergleichen, wie hoch das BIP und die Lebenserwartung in der Schweiz, Deutschland und den USA in den Jahren 2012, 2013 und 2014 waren, dann handelt es sich um TSCS-Daten: Bestimmte Variablen werden für bestimmte Einheiten für unterschiedliche Zeitpunkte erfasst. Die Struktur von TSCS-Daten als wiederholter Querschnittserhebungen ist in formaler Hinsicht identisch mit Panel-Daten, obschon bei Panel-Erhebungen die Anzahl der Zeitpunkte tendenziell geringer als bei TSCS-Daten ist (Beck 2006).

Die Modellierungen sind in der Statistikumgebung R durchgeführt (R Core Team 2015). Für die TSCS-Zeitreihen verwende ich zusätzlich das Paket «plm» (Croissant und Millo 2008) innerhalb der Statistikumgebung R. Bei der Berechnung der TSCS-Zeitreihen implementiere ich die von Beck und Katz (1995, 2011) vorgeschlagenen panel-korrigierten Standardfehler, damit die Effekte in den Modellen nicht überschätzt werden. Die inhaltsanalytisch erfassten Daten sind Zählvariablen; wenn eine Untersuchungseinheit einer deduktiven (Politikfeld) und induktiven (konkretes Thema) Kategorie zugeteilt wird, dann erhöht sich die Anzahl dieser Kategorien innerhalb der Erhebungseinheit um je eins. Zählvariablen als abhängige Variablen sind für OLS-Regressionsmodelle aber suboptimal; stattdessen sollten Regressionsmodelle verwendet werden, denen passendere Verteilungsannahmen zugrunde liegen, etwa Poisson- und NegativBinomial-Modelle (Cameron und Trivedi 2013, S. 71-88). Das Problem bei diesen Modellen für Zählvariablen ist, dass für deren Umsetzung als Zeitreihe die Berechnung der panel-korrigierten Standardfehler nicht ohne Weiteres möglich ist. Aus diesem Grund verzichte ich auf die für Zählvariablen optimierten Modelle. Stattdessen transformiere ich die Zählvariablen zu relativen Anteilen, wie dies z.B. Brandenburg (2002) vorschlägt. Die Variablen der Politikfelder und der konkreten Themen kommen in einer Erhebungseinheit unterschiedlich oft vor. Um diese absoluten Häufigkeiten zu relativen Anteilen pro Erhebungseinheit zu transformieren, genügt eine simple Bruchrechnung. Wenn beispielsweise interessiert, welchen Anteil das Politikfeld Aussenpolitik an der gesamten Berichterstattung des Tages-Anzeiger an einem Tag ausmacht, dann muss schlicht die Anzahl der Kategorie Aussenpolitik in der betroffenen Ausgabe durch die Summe aller Politikfelder (d.h., aller codierten Untersuchungseinheiten, in diesem Beispiel aller Artikel) geteilt werden. Mit demselben einfachen Vorgehen lassen sich die absoluten Häufigkeiten der konkreten Themen zu den relativen Anteilen transformieren. 


\subsubsection{Auswertung der Interviews}

Die Auswertung der Interview-Transkripte erfolgt qualitativ mit dem Ziel, in den Transkripten induktive Kategorien zu den einzelnen in Unterunterabschnitt 4.2.2 beschriebenen Themenblöcken herauszuarbeiten. Um diesen Arbeitsablauf übersichtlich zu halten, habe ich die Software MaxQDA (Kuckartz 2015) verwendet. Die Auswertung der Interviews ist somit de facto eine qualitative Inhaltsanalyse, und zwar eine, nach Mayring (2010), strukturierende Inhaltsaalyse.

\subsubsection{Zusammenführen der Ergebnisse}

Der Designansatz der Triangulation beschreibt, wie in Unterabschnitt 4.1 dargelegt, nicht ein einziges konkretes Forschungsdesign, sondern meint eher eine bestimmte erkenntnistheoretische Herangehensweise an empirische Forschung. Damit können die konkreten Forschungsanlagen, welche im Sinne des Paradigmas der Triangulation aufgestellt werden, eine grosse Vielfalt aufweisen. Sie haben aber alle eine Gemeinsamkeit: Triangulation fördert im Mindesten zwei separate empirische Ergebnisse zutage. Das Ziel von Triangulation ist aber nicht, disparate Empirie herzustellen, sondern, einen Untersuchungsgegenstand aus mehreren Winkeln auszuleuchten. Darum ist es unumgänglich, die mittels triangulierenden Verfahren gewonnenen empirischen Ergebnisse zu einem empirischen Gesamtbild zusammenzuführen.

Eine wichtige Entscheidung in diesem Zusammenhang ist, wie Jick (1979) bemerkt, die Gewichtung der einzelnen Ergebnisse. In Unterabschnitt 4.1 erkläre ich, dass ich die Inhaltsanalyse und die teilstrukturierten Interviews als gleichwertig erachte. Folglich gewichte ich auch die Ergebnisse der Datenauswertung nicht unterschiedlich, sondern erachte sie auch in dieser Hinsicht als gleichwertig.

Eine prinzipielle Gleichwertigkeit der empirischen Ergebnisse gibt für sich genommen aber noch nicht vor, wie genau die Ergebnisse miteinander zu verbinden sind. O’Cathain, Murphy und Nicholl (2010) beschreiben einige Vorgehensweisen für das Verbinden der Ergebnisse bei Mixed Methods-Studien in medizinischen Kontexten. Wenn auch die Autoren das wichtige Argument nennen, dass das Zusammenführen der Ergebnisse das eigentliche Triangulieren darstellt, auf dessen Grundlage die Interpretation der Ergebnisse stattfindet, bleiben die Vorschläge für das Zusammenführen der Daten eher allgemeiner Natur. Dies aus dem Grund, dass es keine formalen Prozeduren gibt, um triangulierte Ergebnisse zu einer einzigen Aussage zu vermengen, wie dies beispielsweise bei Metaanalysen möglich ist. Moran-Ellis u. a. (2006) unterscheiden zwei Formen der Zusammenführung der Ergebnisse, «seperate methods, integrated analysis» und «separate methods, separate analysis, theoretical integration». 
Tabelle 11: Methoden zur Prüfung der Hypothesen.

\begin{tabular}{ll}
\hline Hypothese & Methoden \\
\hline H1 & Inhaltsanalyse + Interviews \\
H2 & Inhaltsanalyse + Interviews \\
H3 & Inhaltsanalyse + Interviews \\
H4 & Inhaltsanalyse + Interviews \\
H5 & Inhaltsanalyse + Interviews \\
H6 & Inhaltsanalyse + Interviews \\
H7 & Interviews \\
H8 & Inhaltsanalyse \\
H9 & Inhaltsanalyse + Interviews \\
\hline
\end{tabular}

Bemerkung: Hypothese H7 wird lediglich anhand der Interview-Daten geprüft, Hypothese H8 lediglich anhand der inhaltsanalytischen Daten. Alle anderen Hypothesen werden trianguliert durch eine gleichgewichtete Betrachtung sowohl der Interview-, wie auch der inhaltsanalytischen Daten.

Die Ergebnisse der Triangulation für die vorliegende Arbeit führe ich in der letzteren Variante zusammen: Die Datenauswertung führe ich separat für die inhaltsanalytischen und die interviewbasierten Daten durch und führe erst die Ergebnisse zusammen. Die erste Variante der «integrierten Analyse» meint den Vorschlag, die trianguliert erhobenen Daten gleichzeitig in quasi-explorativer Manier auszuwerten; ein nur leidlich systematischer und kaum reproduzierbarer Vorgang. Da ich die Datenanalysen separat durchführe, besteht die einzige Möglichkeit, sie zusammenzuführen, darin, die Ergebnisse argumentativ zu verbinden und zu reflektieren. Ein leicht verkomplizierender Aspekt in dieser Hinsicht ist der Umstand, dass ich nicht für alle in Unterabschnitt 3.4 formulierten Hypothesen trianguliere. Tabelle 11 fasst zusammen, welche Hypothesen mit welchen Methoden geprüft werden.

Die Hypothesen H7 und H8 werden nicht trianguliert, sondern nur anhand der Interviews bzw. anhand der Inhaltsanalyse geprüft. Das ist nicht mangelnden oder mangelhafen Daten geschuldet, sondern der Natur der Hypothesen. Die Hypothese $\mathrm{H} 7^{33}$ spricht die Motivstruktur der Parlamentarierinnen und Parlamentarier an, welche sich nicht aus den inhaltsanalytisch erfassten Daten ablesen lässt. Für Hypothese $\mathrm{H} 8^{34}$ ist das Gegenteil der Fall, denn sie betrifft die Häufigkeiten, mit denen Journalistinnen und Journalisten über die unterschiedlichen Teilarenen der Bundesversammlung berichten; diese Grössen lassen sich zuverlässig aus den inhaltsanalytischen Daten ablesen.

33 «Parlamentarierinnen und Parlamentarier handeln medialisiert als Mittel zum Zweck zugunsten der Politiklogiken».

34 «Medien berichten am meisten über die traktandierten Geschäfte, weniger über die eingereichten Vorstösse und am wenigsten über die Fragestunden im Nationalrat». 


\subsection{Schwächen des Designs und der Methoden}

Das Forschungsdesign ist, funktional gesehen, Mittel zum Zweck der Beantwortung der Forschungsfragen. Die Aussagekraft der Ergebnisse, welche über die Erhebung und Auswertung der Daten zutage gefördert werden, lässt sich denn auch nur einschätzen, wenn auch die Schwächen des Designs bedacht werden. Das Forschungsdesign gibt in diesem Sinne Aufschluss darüber, in welchem Masse das, was untersucht werden sollte, mit dem, was effektiv untersucht wurde, korrespondiert.

Das Design der vorliegenden Arbeit hat in Betreff der analysierten Stichproben einen bedeutenden Mangel, welcher die Aussagekraft der Ergebnisse mindert. Bei der Inhaltsanalyse erfasse ich die gesamten parlamentarischen Aktivitäten der drei Sessionen, aber nicht die gesamten Aktivitäten der Medien in diesem Zeitraum. Nun macht es natürlich Sinn, nicht alle Medien und alle Medienberichterstattung während der Sessionen zu untersuchen, sondern nur eine Stichprobe. Das Problem mit der gewählten Stichprobe an Medientiteln ist aber, dass ich ausschliesslich Deutschschweizer Medien beachte. Das schafft eine deutliche Asymmetrie: Für die parlamentarischen Aktivitäten während der Sessionen mache ich eine Totalerhebung, und zwar nicht zuletzt, weil eine sprachregionale Trennung innerhalb der Sessionsaktivitäten sinnfrei wäre. Wenn auf Medienseite aber nur Deutschschweizer Titel beachtet werden, ist die damit implizierte Annahme, dass eine Deutschschweizer Stichprobe an Medien als pars pro toto auch für Medien aus der Romandie und dem Tessin gelten kann. Auch wenn diese Annahme empirischen Gehalt haben sollte - wofür ich keine Evidenz vorzuweisen habe - , bleiben die asymmetrischen Daten aus einer handlungstheoretischen Perspektive ein Problem. Es ist nämlich anzunehmen, dass die Interaktionen zwischen Parlamentarierinnen und Parlamentariern und Journalistinnen und Journalisten intensiver innerhalb der Sprachgruppen als zwischen den Sprachgruppen sind. Das bedeutet konkret, dass Deutschschweizer Parlamentarierinnen und Parlamentarier stärker mit Deutschschweizer Journalistinnen und Journalisten interagieren, Westschweizer Parlamentarierinnen und Parlamentarier stärker mit Westschweizer Journalistinnen und Journalisten, Tessiner Parlamentarierinnen und Parlamentarier stärker mit Tessiner Journalistinnen und Journalisten. Diesen Umstand der sprachlichen Segmentierung hat ein Journalist in einem der Interviews ebenfalls angesprochen, als er zu beschreiben versuchte, nach welchen allgemeinen Logiken und mit was für Heuristiken Medienschaffende ihrer Arbeit nachgehen:

Ich würde Mal so sagen, in der Westschweiz kennt man die wenigsten Parlamentarier aus der Deutschschweiz. [...] Weshalb? Weil die Medien natürlich immer 
diejenigen Leute nehmen, die aus ihrem Sprachgebiet kommen. Ist ganz interessant, oder. Unsere Westschweizer Kollegen [...], die nehmen natürlich ihre Leute aus der Westschweiz. [...] Deshalb gibts zum Teil auch eine etwas, eine mediale Verzerrung.

Wenn nun eine Asymmetrie zwischen der Totalerhebung der Parlamentsaktivität und der sprachregional begrenzten Stichprobe der Medien besteht, bedeutet das mit hoher Wahrscheinlichkeit, dass die quantitativ gemessenen Agenda Setting-Effekte unvollständig abgebildet werden, weil ein Teil der Wechselwirkungen mit Medien aus den anderen Sprachregionen stattfindet. Das hat zwangsläufig zur Folge, dass die quantitativ gemessenen Effekte tendenziell zu schwach sind. Bei der Auswahl der Interviewpartnerinnen und -partner habe ich mich ebenfalls nur auf Deutschschweizer Mitglieder des National- und Ständerates sowie auf Deutschschweizer Journalistinnen und Journalisten beschränkt. Auch hier stellt sich die generelle Frage, ob die Erfahrungen und Einschätzung dieser Deutschschweizer Stichprobe eine generalisierbare Aussagekraft besitzen. Das Problem ist aber auf der rein analytischen Ebene nicht akzentuiert wie bei der Inhaltsanalyse, weil die sprachregionale Zugehörigkeit der zwei Gruppen dieselbe ist. Eine ähnlich problematische Konfiguration wie bei der Inhaltsanalyse wäre es gewesen, die Stichprobe an Parlamentarierinnen und Parlamentariern so zu belassen, wie sie ist, die Stichprobe an Journalistinnen und Journalisten aber auf die Romandie und das Tessin auszuweiten.

Das bedeutet aber nicht, dass die Interviews grundsätzlich unproblematisch sind. Qualitative Forschung sieht sich, im Allgemeinen, mit der Grundsatzproblematik konfrontiert, dass die Trennlinie zwischen qualitativen Daten und blossen Anekdoten nicht immer sehr klar ist (Merriam 1995). Bei der Methode der qualitativen Interviews, im Besonderen, kommen noch weitere erschwerende Umstände hinzu, allen voran vielleicht die Reaktivität der Methode (Onwuegbuzie und Leech 2006; Roulston 2010). Diese zwei Gefahren werden im Rahmen des verwendeten Forschungsdesigns in zweierlei Hinsicht eingedämmt. Erstens ist es genau die Stärke eines triangulierenden Designs, die Schwachpunkte einer bestimmten methodischen Herangehensweise durch das Verwenden mindestens einer zweiten methodischen Herangehensweise aktiv zu kompensieren. Zweitens verträgt sich meine erkenntnistheoretische Ambition der durchgeführten Interviews mit der erkenntnistheoretischen Güte von Interviews als Methode. Bei den durchgeführten Interviews handelt sich nämlich, wie in Unterunterabschnitt 4.2.2 beschrieben, um Experteninterviews, und das Ziel der Interviews ist es, Handlungsdynamiken und Motivstrukturen von Akteuren innerhalb dieser überschaubaren Teilsysteme zu erkunden. Das bedeutet, dass die Akteure als Angehörige sehr spezifischer Teilsysteme Auskunft geben über ihr Handeln in ihrer Rolle als Mitglieder dieser sehr spezifischen Teilsysteme. 
Nebst diesen gewichtigeren Aspekten sind einzelne weitere Details im Rahmen des Forschungsdesigns suboptimal, welche aber nicht in einem Masse schwerwiegend sind, dass wegen ihnen das Design an und für sich in Frage gestellt würde. So habe ich ein Teil der Interviews nicht auf Standarddeutsch, sondern auf Schweizerdeutschem Mundart geführt. Das hat einerseits einen leichtem Informationsverlust bei der Transkription der Interviews zur Folge, da das Mundart auf Standarddeutsch «übersetzt» wird. Andererseits, und vielleicht wichtiger, ist der Umstand, dass das individuelle Denken und Empfinden durch die Sprache, welche verwendet wird, geprägt ist - egal, ob Schweizerdeutsch und Standarddeutsch als gleichwertige Sprachen bei bi- oder multilingualen Individuen betrachtet werden (Luna, Ringberg und Peracchio 2008), oder, ob Standarddeutsch als Fremdsprache betrachtet wird (Keysar, Hayakawa und An 2012). Somit ist es etwa möglich, dass das Sprechen auf Standarddeutsch eine systematische Verzerrung bedeutet. Diese Möglichkeit kann ich nicht ausschliessen, aber ich gehe davon aus, dass der verzerrende Effekt, so er existiert, eher klein ist.

Ein weiterer Aspekt, welcher nicht optimal gelöst ist, betrifft die Granularität der Inhaltsanalyse. Wie in Unterunterabschnitt 4.2.1 festgehalten ist, hat die aufwendige qualitative Inhaltsanalyse rund 1'000 unterschiedliche konkrete Themen pro Stichprobenjahr zutage gefördert. Für die quantitative Auswertung dieser qualitativen Daten interessieren aber nicht individuelle Themenkarrieren, sondern aggregiert beobachtbare Effekte. Es wäre darum an und für sich angebracht gewesen, einen weiteren Triangulationsschritt einzuführen: Die qualitative Rekonstruktion einzelner Themenkarrieren. Ein solcher Triangulationsschritt würde eine empirische Brücke zwischen generellen, quantitativ gemessenen Effekten und den individuellqualitativen Einschätzungen der betroffenen Akteure schlagen. Hier spielt aber schlicht das Nullsummenspiel der verfügbaren Ressourcen eine Rolle: Je mehr Triangulationsschritte mit den endlichen für die Forschung verfügbaren Ressourcen durchgeführt werden, desto oberflächlicher fallen die Triangulationsschritte aus. Die Anzahl Triangulationsschritte ist also ein Abwägen von Breite gegen Tiefe der Triangulation - für die vorliegende Arbeit habe ich mich für mehr Tiefe entschieden.

Open Access Dieses Buch wird unter der Creative Commons Namensnennung - Nicht kommerziell 4.0 International Lizenz (http://creativecommons.org/licenses/by-nc/4.0/deed. de) veröffentlicht, welche für nicht kommerzielle Zwecke die Nutzung, Vervielfältigung, Bearbeitung, Verbreitung und Wiedergabe in jeglichem Medium und Format erlaubt, sofern Sie den/die ursprünglichen Autor(en) und die Quelle ordnungsgemäß nennen, einen Link zur Creative Commons Lizenz beifügen und angeben, ob Änderungen vorgenommen wurden.

Etwaige Abbildungen oder sonstiges Drittmaterial unterliegen ebenfalls der genannten Creative Commons Lizenz, sofern sich aus der Abbildungslegende oder der Quellreferenz nichts anderes ergibt. Sofern solches Drittmaterial nicht unter der genannten Creative Commons Lizenz steht, ist eine Vervielfältigung, Bearbeitung oder öffentliche Wiedergabe nur mit vorheriger Zustimmung des betreffenden Rechteinhabers oder auf der Grundlage einschlägiger gesetzlicher Erlaubnisvorschriften zulässig. 